\title{
CFD based analysis of flow-driven vertical axis hydrokinetic turbines: blade hydrofoil and interactions effects
}

\author{
Zaid Hammoudi ${ }^{1}$, Ikram El Abbassi ${ }^{1,2}$, Rafik Absi ${ }^{1,3, *}$, A.-Moumen Darcherif ${ }^{1,3}$ \\ ${ }^{1}$ ECAM-EPMI Graduate School of Engineering, LR2E-Lab, 13 Boulevard de l'Hautil, 95092 Cergy-Pontoise (France) \\ ${ }^{2}$ L2MGC-Lab - EA 4114. ${ }^{3}$ Quartz-Lab - EA 7393,
}

\begin{abstract}
In last years, interest in hydrokinetic energy conversion (HEC) technology has grown. However, HEC technology requires advancements to become successful for practical in-situ conditions. In this study we investigated the Darrieus type vertical axis hydrokinetic turbines (HKT). Simulations are carried out using commercial CFD package to study the hydrodynamic behavior of a three bladed turbine. Two HKT with same solidity equal to 0.17 were considered, a 1 st with symmetric-NACA0018 blade hydrofoils and a 2nd with cambered-NACA4415 blades. Our simulations show a shift in instantaneous torque coefficients for two different tip-speed ratio (TSR) values. For TSR $=1$, the 2 nd HKT has a delay compared to the 1 st HKT, while for TSR $=2$ it has a lead. NACA0018 provides highest troque coefficients. For a hydrofarm use, it is important to consider the interactions between hydrokinetic turbines. We investigated the effect of streamwise distance on performance of a Darrieus turbine. A distance of about 11 times the diameter of HKT along the streamwise direction seems essential.
\end{abstract}

\section{Introduction}

Fuels and nuclear power represents more than $80 \%$ of the energy used in the world. These sources are easy to exploit, but the major problem is that they are not inexhaustible, and also cause environmental problems, such as greenhouse effect and nuclear waste. Most countries have already begun a transition to clean, renewable energy for their future. Non-conventional renewable energy (NCRE) sources can be classified into five families: solar, wind, biomass, geothermal and hydro or hydroelectric. In last years, interest in hydrokinetic energy conversion (HEC) technology has grown. However, hydrokinetic energy from tidal and river flows is currently little exploited around the world but generates great expectations due to its high potential for sustainable and predictable energy. The potential for total tidal energy in the world is estimated at about 3 $\mathrm{TW}$, including $1 \mathrm{TW}$ in accessible areas for the installation of energy capture devices [1]. To capture this kinetic energy, more than 50 devices can be used [2], in particular machines derived from wind turbine technologies, called tidal turbines [3] or hydrokinetic turbines (HKT), immersed along coasts or in rivers, or suspended on the free surface of the water [4].

There are mainly two types of turbines according to the position of the axis of rotation with respect to the current: horizontal axis (axial-flow) HKT and vertical axis (cross-flow) HKT. Darrieus type cross-flow hydrokinetic turbines (HKT) has some advantages over axial-flow turbines [4]. Different studies have been conducted to better understand cross flow hydrokinetic turbines HKT [5-7]. HEC technology requires advancements to become successful for practical applications. We need better understanding (1) individual turbines to allow optimization and (2) turbine wake and HKT interactions effects. Thanks to rapidly increasing computing power, Computational Fluid Dynamics (CFD) has become a powerful tool in engineering design and optimization. With adequate numerical methods, CFD provides accurate, cheap and quick results compared to a full-scale experimental study [8-9]. The aim of our study is to use CFD in order to provide an accurate description of the flow and therefore the best conditions for an optimization. Different commercial CFD package can be used, such as ANSYS CFX and Fluent [5-6]. In this study, we will use ANSYS Fluent for simulations of vertical turbines. We will introduce background related to HKT, define the different parameters and more adequate turbulence models. Finally, simulations with different parameters, geometries and configurations will be conducted.

\section{Hydrokinetic turbines (HKT)}

\subsection{Principle}

Hydrokinetic turbines are similar to wind turbines, they use the kinetic energy of flowing water to drive a generator [11]. However, the main difference is about the density of water which is about 850 times greater than air and flow velocities for a tidal or river flow tend to be an order of magnitude lower than flow velocities of

\footnotetext{
*Corresponding author: r.absi@ecam-epmi.com
} 
wind. Finaly, the Reynolds numbers are in the same range for both hydrokinetic and wind turbines.

Hydrokinetic turbines are on seabed or suspended under the free surface of the water. The rotor of the turbine turns under the effect of the current then drives an alternator that transforms mechanical energy into electrical energy.

\subsection{Maximum extracted power: Betz's Law}

The first efforts to assess the performance of rotating machines under the effect of a fluid flow (i.e. wind turbines) were done by [11] and [12]. The well know Betz's or Lanchester-Betz's law, which defines the maximum power that can be extracted from the stream flowing through an idealized « actuator disk » of section S. From the principles of conservation of mass and momentum, the power extracted from the water through the rotor is given by $[11,12]$ :

$P=\frac{1}{2}(\rho S v)\left(v_{1}^{2}-v_{2}^{2}\right)$

where : $v_{1}$ et $v_{2}$ are respectively velocities in the front and downstream of the rotor, $\rho$ the water density and $v=\frac{1}{2}\left(v_{1}+v_{2}\right)$.

The totale power of a non-perturbed flow without rotor as for a cylinder of fluid with cross sectional area $\mathrm{S}$ is:

$P_{0}=\frac{1}{2} \rho S v_{1}^{3}$

The power coefficient is given by :

$C_{p}=\frac{P}{P_{0}}=\frac{1}{2}\left(1-\left(\frac{v_{2}}{v_{1}}\right)^{2}\right)\left(1+\left(\frac{v_{2}}{v_{1}}\right)\right)$

The curve of equation (3) riches a maximum for $v_{2} / v_{1}=1 / 3$, the maximale value is $C_{p}=P / P_{0}=16 / 27$. According to Eq. (3), no turbine can capture more than $16 / 27(59.3 \%)$ of the kinetic energy of the flow (Ahmadi-Baloutaki et al. 2015). The factor 16/27 (0.593) is known as Betz's or LanchesterBetz's coefficient. Practical utility-scale wind turbines achieve at peak $75 \%$ to $80 \%$ of the Lanchester-Betz's limit.

\subsection{Tip Speed Ratio}

The tip-speed ratio, or TSR for turbines is the ratio between the tangential speed of the tip of a blade and the actual flow velocity:

$T S R=\lambda=\frac{\omega R}{v_{\infty}}$

where $\omega$ is the rotor rotational speed in radians/second, $R$ the rotor radius in meters et $v_{\infty}$ is the flow velocity at an infinite point from the turbine in meters/second. The tip-speed ratio is related to turbines efficiency.

\subsection{Power and torque coefficients}

The torque coefficient is given by:

$C_{M}=\frac{M_{Z}}{\frac{1}{2} \rho(H \phi) R v_{\infty}^{2}}$

where $M_{z}$ the torque along the axis of rotation $\overrightarrow{O Z}, S=$ $H \phi, H$ and $\phi$ are respectively the high and the diameter of the hydrokinetic turbine.

The power coefficient $C_{P}$ is the ratio between the power drawn from the water by the hydrokinetic turbine and the power of the flow:

$C_{P}=\frac{P}{\frac{1}{2} \rho(H \phi) v_{\infty}^{3}}$

It is also possible to write the following relationship between power and torque:

$P=M_{z} \omega$

From the previous relationships, we can deduce that:

$C_{P}=\lambda C_{M}$

\section{Results and discussions}

We used statistical Reynolds Averaged Navier-Stokes (RANS) turbulence models. RANS models offer the most economic approach for computing turbulent industrial flows. Examples of RANS models are the different forms of $k-\varepsilon$ or the $k-\omega$ two-equation models $[13,14]$. These models are based on the concept of eddy viscosity (turbulent viscosity) which is obtained by solving two additional transport equations. RANS models are suitable for many engineering applications and typically provide the level of accuracy required. Among the RANS models, the two-equation eddyviscosity shear stress transport (SST) $k-\omega$ turbulence model combines a $\mathrm{k}-\omega$ formulation in the inner parts of the boundary layer and a k- $\varepsilon$ model a far from the wall [15]. In the present study, the flow is modeled using the k- $\omega$ SST model available in ANSYS Fluent.

To validate our method and results, we consider the cross-flow hydrokinetic turbine of Darrieus type of LEGI Laboratory (Grenoble). This HKT was used in the hydrodynamic tunnel of LEGI which consists in a rectangular section of $0.25 \times 0.7 \mathrm{~m}$ and a $1 \mathrm{~m}$ length in the streamwise direction inserted in a closed hydraulic loop of $30 \mathrm{~m}$. Flow velocity are between 1 to $2.3 \mathrm{~m} / \mathrm{s}$. It corresponds to a blade Reynolds number between $1.7 \times 10^{5}$ and $5 \times 10^{5}$. Rotational speed is imposed by a synchronous generator connected to the turbine shaft. Turbine has 3 NACA0018 blade hydrofoils. The height $\mathrm{H}$ and the diameter $\mathrm{D}$ of the turbine are equal, $\mathrm{H}=\Phi=175 \mathrm{~mm}$ and blade chord $\mathrm{C}=32 \mathrm{~mm}$ (figure 1 . a) The solidity which is the fraction of circumference of rotor covered by blades is equal thereofore to 0.17 . It represents a lateral blockage ratio (tunnel width/turbine width) of 4 , and a vertical blockage ratio of 1.43 . 
It is important to select first the most appropriate mesh. Validation of our first results is done by comparisons with those of [6]. We will create the geometry with DesignModeler and mesh with Meshing. The methodology is explained in the next section.
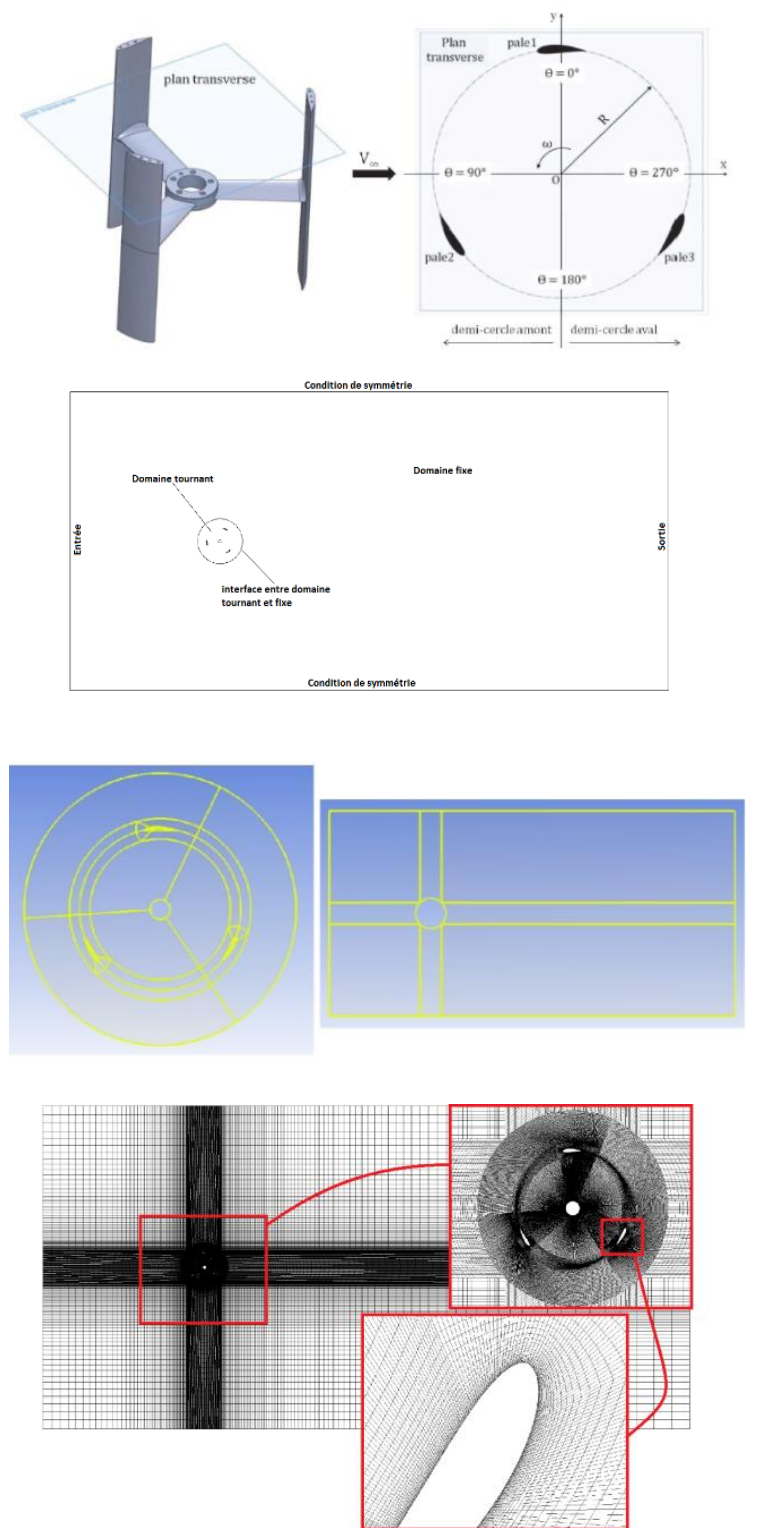

Fig. 1. Geometry and mesh, (Top) 3D view of the hydrokinetic turbine and its 2D section used for calculations, (Menchaca Roa 2011) (2nd) Geometry of a vertical hydrokinetic stream in an infinite medium, (3rd) Geometry under ANSYS Fluent, (Bottom) Structured mesh.

\subsection{Geometry}

The geometry is composed by two parts: 1) the first is rotating, of circular section with the horizontal sections of the three blades of the hydrokinetic turbine and its axis, 2) the second is fixed representing the medium surrounding the hydrokinetic turbine. Between the two, we apply a sliding mesh interface.

- The hydrokinetic turbine used has a diameter $\Phi=(2 \mathrm{R})=0.175 \mathrm{~m}$, an axis of diameter $\mathrm{d}=$
$0.022 \mathrm{~m}$, a height $\mathrm{h}=0.175 \mathrm{~m}$, a blade chord $\mathrm{C}$ $=0.032 \mathrm{~m}$ and it is possible to change the shape of blade hydrofoil. The geometry is done with Design Modeler, the procedure is as follows:

- We import the coordinates of points constituting the blade hydrofoil NACAxxxx, then we transform them into a curve and apply functions "transformation of body" to move it to the desired place and duplicate it in a circular way to have the three blades.

- We draw all the other sketches of the geometry, sharing the two parts rotating and fixed in several surfaces to create several geometry bodies (Figure 1.c) to make a structured mesh, knowing that each part is created separately.

- $\quad$ At the end, we can gather the two pieces in one. By importing them, the piece fixes as a blocked body, and the rotating part as an unlocked body to apply fluent the sliding mesh technic and give the desired rotation.

\subsection{Mesh}

The mesh is made using Meshing software integrated in Ansys Workbench. Geometry already shared in geometry bodies. We create sizing on each edge, inflations around the walls of the blades and the axis of rotation and we apply the meshing method of faces to create a mesh in quadrilaterals (figure 1.d). Finally, we add the named selections for boundaries and the interface between rotating and fixed part [16].

Once the mesh created, we import it under Fluent and adjust the various parameters before starting the computation: First to create an interface between the rotating part and the fixed part: "Mesh Interface".

Then we can proceed as:

- Unsteady regime,

- We choose the turbulence model, $k-\omega$ (2eqn) in "Model" and SST in " $k-\omega$ model".

- In "Cell Zone Conditions", we put the desired rotation for the rotating zone by checking the "Mesh Motion" box and choose the axis of rotation and the angular velocity.

- We Define boundary conditions in "Boundary Conditions": Choose "symmetry" for the two horizontal boundaries of the domain. We choose "wall" for the three blades. For velocity inlet, velocity $=2.3 \mathrm{~m} / \mathrm{s}$, turbulence intensity $\mathrm{I}=3 \%$, characteristic length $1=$ 0.002 as turbulence quantities. They were chosen by analogy with smooth pipes, since no experimental data are available [6]. Finally, we take for output "pressure outlet", the same parameters of turbulence as input.

- For computation of torque coefficient $C_{M}$ given by equation (5), Fluent uses reference values, which must be inserted in "reference values", these values are the cross-sectional surface, the water density, the hydrokinetic turbine depth, a reference length, a reference velocity and the dynamic viscosity of water.

- We choose the resolution method,

- We set the residue values at $10^{-5}$.

- And finally, before starting simulations, we define the time step, which is chosen as a degree of rotation [6], 
to obtain the best compromise between precision and computational time, we increased the number of step times to reach a periodic solution with $100 \max$ iterations per time step.

To obtain the better computation results, we need a mesh well adapted to the problem geometry. At initial step we used 3 different meshes: $1^{\text {st }}$ mesh is unstructured, the $2^{\text {nd }}$ is structured in part, the $3^{\text {rd }}$ is structured and finer than the two others (about 180,000 points). We took this $3^{\text {rd }}$ mesh for the simulations since the results at TSR $=2$ show a good agreement with that of [6].

As shown in equation (8), to know the power factor $C_{P}$, we first need to compute the torque coefficient $C_{M}$, which is obtained by taking the average of instantaneous torque coefficient over a complete period. However, computations must be left until a periodic solution is obtained, as shown in FIG. 2. FIG. 3 presents instantaneous torque coefficients of the hydrokinetic turbine or of each of the individual bladesare.

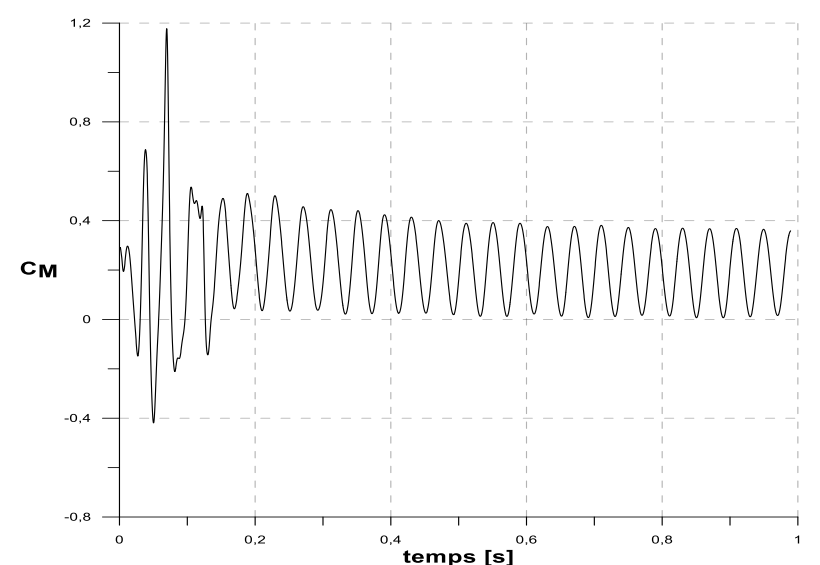

Fig. 2. Torque coefficient for the hydrokinetic turbine.

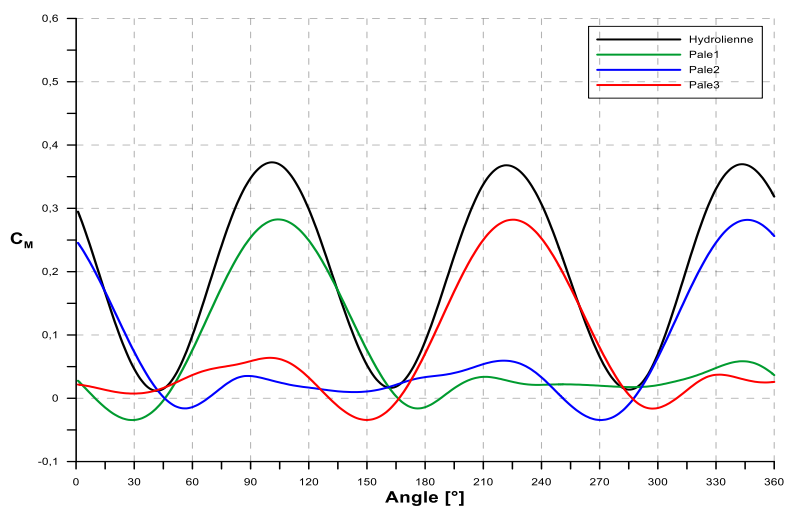

Fig. 3. Torque coefficient with $\mathrm{TSR}=2$.

\subsection{Simulations with NACA4415 blade hydrofoil}

After validating the simulations and the used mesh, the aim of this part is to investigate the effect of blade hydrofoil on the performance of the cross-flow hydrokinetic turbine of Darrieus type. We changed the symmetric-NACA0018 hydrofoils by cambered-NACA4415 blades to compare the performance of these two types of blade hydrofoils. When hydrokinetic turbines with fixed pitch blades show poor starting torque, it is possible to improve it by cambered blade profiles [4]. The simulation conditions are the same. Two simulations were carried out with $\mathrm{TSR}=1$ and $\mathrm{TSR}=2$.
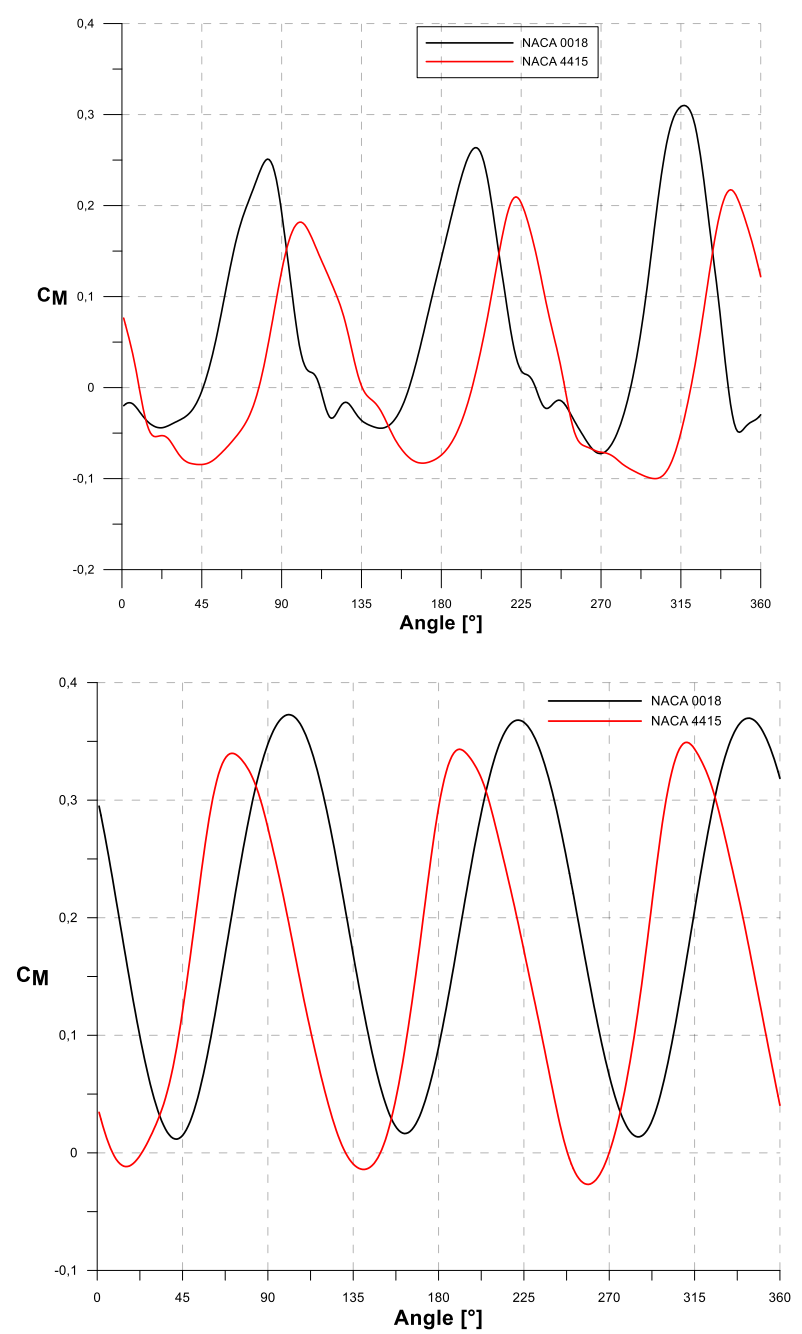

Fig. 4. Instantaneous torque coefficients: (Top) $T S R=1$, (Botton) TSR=2.

Figure 4 illustrates the difference in instantaneous torque coefficient for the two cases, between the hydrokinetic turbine (1) with NACA0018 blades and the hydrokinetic turbine (2) with NACA4415 blades. NACA0018 provides highest torque coefficients for the two TSR values and therefore a higher Power coefficient. However, we notice a shift of the hydrokinetic turbine (2) compared to the hydrokinetic turbine (1), it has a delay for $\mathrm{TSR}=1$, while for TSR $=2$ it has a lead. The torque and therefore the power is more important for the hydrokinetic turbine (1), which gives a greater depression between the intrados and the extrados of the blades, thus a greater lift force, as well as a greater tangential force.

\subsection{Effect of streamwise distance between Darrieus turbines toward hydro farm design}

In marine environment, HKT are implanted in farms, such as wind turbines. The main shortcoming of HKT is the wake they create, which affects the power of other 
turbines downstream. The simple actuator disc model is unable to provide an adequate description. It can't reproduice blade tip vortices and wake rotation which provide a strong contribution to turbulence. Zhang et al. investigated near-wake wind turbine by PIV measurements [17]. They noticed a dislocation of coherent vortices after a few diameters and the important impact on turbulence production.

Figure 5 shows the change in the turbulence intensity caused by a single HKT. It is important to accurately predicte turblence quantities downstream a rotor since it is involved in the flow energy available for a 2 nd HKT in hydrofarm configurations.

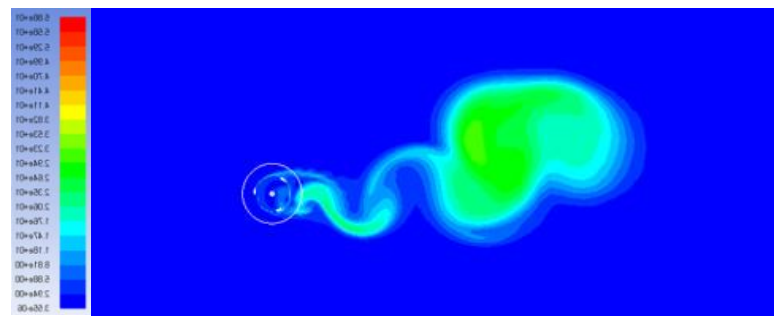

Fig. 5. Turbulence intensity (\%).

In the case of two HKT in the streamwise direction, the second at downstream side has weak flow since the power density, which is the amount of power per unit volume, of the flow is less important compared to the 1 st HKT at upstream side. This could be explained by the flow diversion after the 1st HKT due to loss in kinetic energy (Fig. 5). We aim to investigate the required distance for which the flow will recover his entire nonperturbed energy.

To understand the effect of the interaction between HKT, a simulation of two axially aligned turbines (NACA4415) separated by a distance $\mathrm{A}=1 \mathrm{~m}$ and then $2 \mathrm{~m}$, which represents respectively about 6 times and 11 times the diameter of the hydrokinetic turbine.

Figure 6 shows the effect of the first hydrokinetic turbine on the power of the second. The torque coefficient of the 2nd HKT is closer to that of the first one when $X=2 \mathrm{~m}$. For $\mathrm{X}=1 \mathrm{~m}$, we notice a gap between torque coefficients of the two HKT.

Simulations show that the wake generated by upstream HKT has a high influence on the performance of the downstream HKT. The turbulence created by the 1 st HKT dissipates away, so the further the 2nd HKT is distant, the closer their respective torque coefficients are. There is an optimal distance between two turbines axially aligned for which their torque coefficient becomes quite close. A stream wise distance of about $\mathrm{X} / \mathrm{D}=11$ seems to be essential to have the least wake effect.

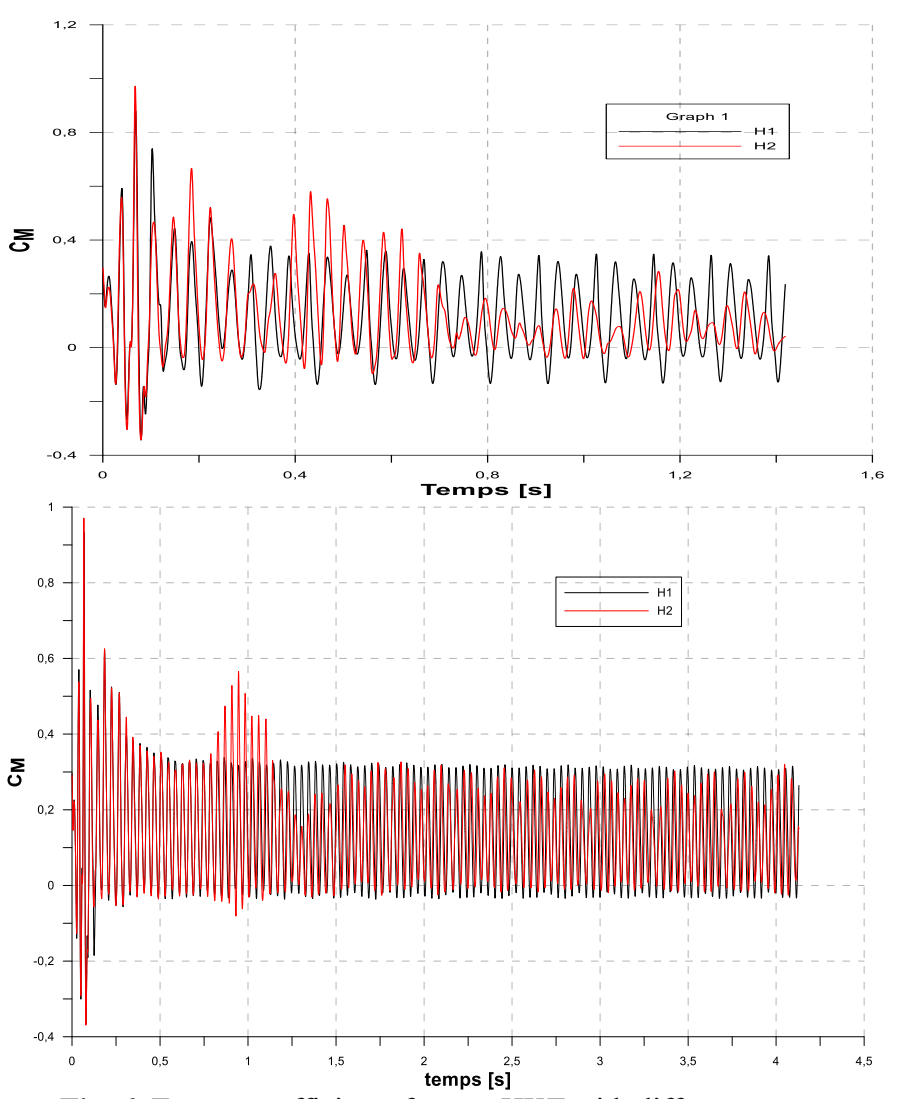

Fig. 6. Torque coefficients for two HKT with different streamwise distances: (top) $\mathrm{X}=1 \mathrm{~m}$ and (Bottom) $\mathrm{X}=2 \mathrm{~m}$, respectively about 6 times and 11 times the diameter of the rotor..

\section{Conclusions}

The objective of the present study is to investigate: (1) the influence of hydrofoils on the performance of a Darrieus-type vertical-axis turbine and (2) the interaction between two axially aligned turbines. We performed two-dimensional simulations to study the behavior of the cross-flow Darrieus turbine. We outlined the steps for creating a geometry and mesh to apply the sliding mesh technique with ANSYS Design Modeler, Meshing, and Fluent.

Simulations were carried out for a HKT (1) with symmetric-NACA0018 and HKT (2) with camberedNACA4415 blades in same conditions with TSR $=1$ then $\mathrm{TSR}=2$. According to our results, HKT (1) with NACA0018 hydrofoils provides highest troque coefficients for the two TSR values and therefore more Power. However, we notice a shift in the instantaneous torque coefficient of the hydrokinetic turbine (2) compared to the hydrokinetic turbine (1), it has a delay for $\mathrm{TSR}=1$, while for TSR $=2$ it has a lead. This result shows the importance of the TSR which can reverse the behavior for a HKT with a given blade hydrofoil. This behavior seems less important than the maximum torque coefficient; however it will require future investigations on possible mechanical or vibrating aspects.

In marine environment, HKT are implanted in farms, such as wind turbines. The main shortcoming of HKT is the wake they create, which impacts the power of other turbines downstream. We studied the case of two HKT 
aligned axially in the streamwise direction, separated by a distance respectively equal to about 6 and 11 times the diameter of the HKT. Simulations show that the wake generated by upstream HKT has a high influence on the performance of the downstream HKT. There is an optimal distance between two turbines axially aligned for which their torque coefficient becomes quite close. A stream wise distance of about $\mathrm{X} / \mathrm{D}=11$ seems to be essential to have the least wake effect.

\section{References}

1. Twidell J., Weir T. Renewable Energy Resources, Chapter 13. Taylor \& Francis, (2006).

2. Edenhofer O., Pichs-Madruga R, Sokona Y, Seybot K., Eickemeier P., Matschoss P., Hansen G., Kadner S., Schlömer S., Zwickel T., Von Stechow C. IPCC Special Report on renewable energy Sources and Climate change Mitigation. Prepared by working Group III of the Intergovernmental Panel on Climate change. (Cambridge University Press, 2011).

3. Behrens S., Griffin D., Hayward J., Hemer M.A., Knight C., MCGarry S., Osman P., Wright J. Ocean renewable energy, 2015-2050: an analysis of ocean energy in Australia. CSIRO. 212 p, (2012).

4. Kirke B.K. and Lazauskas L. Renewable Energy, 36(3), 893-897, (2011).

5. Marsh P., Ranmuthugala D., Penesis I., Thomas G. Renewable Energy, 83, pp 67-77, (2015).

6. Menchaca Roa A. PhD thesis, Université de Grenoble, France, (2011).

7. Patel V., Eldho T.I., Prabhu S.V. International Journal of Marine Energy, 17, 110-135, (2017).

8. Bianchini A., Balduzzi F., Bachant P., Ferrara G., Ferrari L. Energy Conversion and Management, 136, 318-328, (2017).

9. Shaheen M., Abdallah S., Sustainable Energy Technologies and Assessments, 19, 125-135, (2017).

10. Bilgili M., Yasar A., International Journal of Green Energy, 14(12), 1048-1056, (2017).

11. Lanchester F.W., J. Am. Soc. Nav. Eng., 27(2), 509-510, (1915).

12. Betz A., Z., für Das. gesamte Turbinenwes., 26 (1920).

13. Absi R, Tanaka H, Kerlidou L, André A., Proc. 33rd International Conference on Coastal Engineering, 1-6 July 2012, Santander, Spain, 2772-2782, (2012).

14. Absi R., Proc. 27th International Conference on Coastal Engineering (ICCE), July 16-21, 2000, Sydney, Australia, American Society of Civil Engineers, 1268 - 1281, (2000).

15. El Gharbi, N., Absi, R., and Benzaoui, A., J. Appl. Fluid Mech., 5, 63-70, (2012).

16. ANSYS INC (2013). ANSYS Fluent User's Guide, Release 15.0. 2620 p.
17. Zhang W., Markfort C.D., and Fernando P., Experiments in fluids, 52(5), 1219-1235, (2012). 